\title{
Spatial resolution in optical transition radiation beam diagnostics
}

\author{
M. Castellano and V. A. Verzilov \\ Istituto Nazionale di Fisica Nucleare, Laboratori Nazionali di Frascati, P.O. Box 13, I-00044 Frascati, Italy
}

(Received 16 June 1998; published 23 October 1998)

\begin{abstract}
An evaluation of the optical transition radiation (OTR) single particle image dimension is obtained using diffraction theory based on a realistic description of the radiation source. This approach allows the analysis of the effect of the finite size of the emitting screen and of the imaging system. The role of practical experimental conditions in treating the intensity tail problem is estimated. It is shown that, by exploiting the polarization properties of OTR, a considerable enhancement in the spatial resolution can be achieved, which becomes very similar to that of a standard point source. [S1098-4402(98)00017-2]
\end{abstract}

PACS numbers: 41.60.-m, 41.85.Ew, 41.75.Ht

\section{INTRODUCTION}

The application of optical transition radiation (OTR) for charged particle beam diagnostics, suggested initially by Wartski [1] and later developed by Rule and Fiorito $[2,3]$, in the last decade has been exploited at a number of accelerators with beam energies ranging from $1 \mathrm{MeV}$ [4] up to $4 \mathrm{GeV}$ [5]. With modern powerful optical detectors, OTR based devices prove to be compact, reliable and inexpensive, radiation and heat resistant, capable of high time resolution, and cause very little disturbance to the beam because the thickness of targets is only a few microns or less. The technique has already given excellent results and is considered one of the basic diagnostic tools for future projects.

Until recently, certain doubts about the ability of OTR to measure the profile of very high energy beams existed because it was believed that its spatial resolution would decrease with increasing beam energy. The origin of the argument came from the fact that decreasing the "effective" angle of emission $\theta \sim \gamma^{-1}$ ( $\gamma$ is the relativistic factor of the beam) would give a limit of the order of $\lambda \gamma$ in the two-point resolution, due to the wellknown diffraction phenomena. On the other hand, the decrease of the emission angle is strictly related to the linear increase with energy of the transverse extension of the electromagnetic field of the charged particle, often identified as the OTR source. For a wavelength $\lambda$ in the optical domain, the above limiting resolution would render OTR useless for beam profile measurements already at a few $\mathrm{GeV}$.

However, more detailed considerations [3,6,7] show that the spatial resolution is mainly determined by the angular acceptance of the optical system used to detect the radiation rather than by the effective emission angle and does not significantly differ from that due to the standard diffraction limit for this case.

In [6] the first serious analysis of the problem was given, and a correct calculation of the OTR intensity distribution in the image plane was made, in the context of geometrical optics, for a highly relativistic regime.
In contrast to [3], it was shown that, unlike standard diffraction patterns from a point source, OTR images from a single particle have a minimum at the center. In [7] attention was also drawn to the fact that the OTR resolution depends strongly on the way full width at half maximum (FWHM) or rms widths are defined, because of the long energy dependent tail in the intensity distribution, and is influenced by the sensitivity of the detector.

In this paper we try to give a more general and consistent treatment of the phenomenon with the purpose of clarifying those aspects that have so far been left in the shadow.

\section{OTR SOURCE}

Normally, it is mostly the source that defines its image; we therefore found it natural to start our examination by giving attention to the source properties. For OTR this is not trivial, given that the charged particle field diverges at small distances from the particle location.

We start by considering the transition radiation emerging in the backward direction when a charge $q$ moving in vacuum with constant velocity $v$ enters normally into a perfect conductor whose surface coincides with plane $z=0$ (Fig. 1). This problem is well known and details can be found in many books (see, e.g., [8]). Hence we give here only a few formulas that illustrate our conclusions and are useful for further analysis.

The moving charge field can be described by a superposition of plane waves (pseudophotons) whose electric components, transverse to the charge velocity, are

$$
\begin{gathered}
E_{x, y}^{q}(z, x, \omega)=-\frac{4 \pi i q}{v} e^{-i(\omega / v) z} \frac{x_{x, y}}{x^{2}+\alpha^{2}}, \\
\alpha=\frac{\omega}{v \gamma}, \quad x_{x, y}=x\left\{\begin{array}{c}
\cos \psi \\
\sin \psi
\end{array}\right\},
\end{gathered}
$$

where $\omega$ is the radiation frequency and $\psi$ is the angle that vector $x$ makes with the $x$ axis; $x_{x, y}$ can thus be interpreted as the transverse components of the pseudophoton wave vector. The boundary conditions on the conducting 


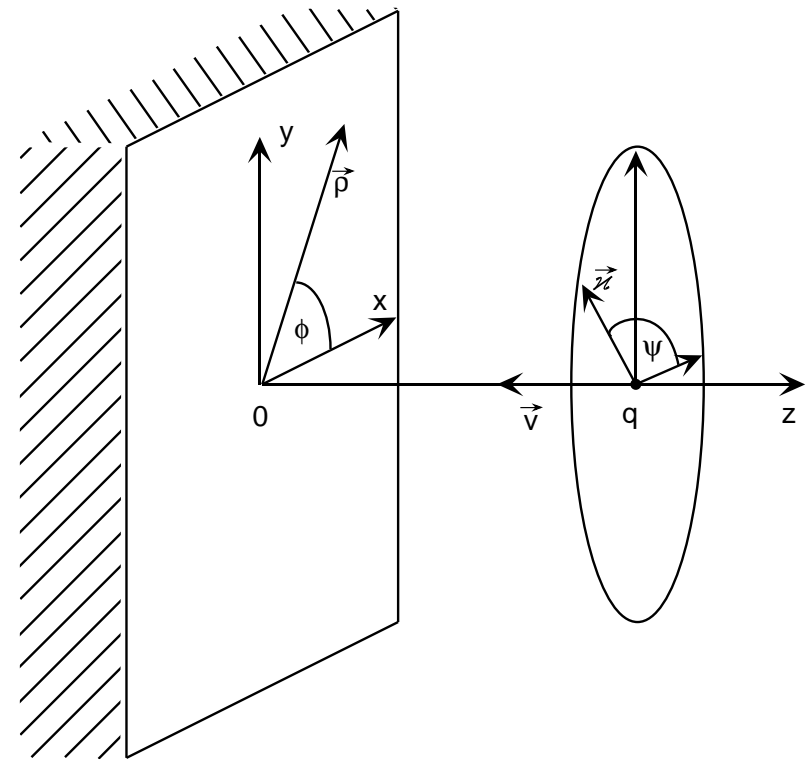

FIG. 1. The moving charge field can be represented by a set of pseudophotons whose wave vector transverse and longitudinal components are $x$ and $\omega / v$, respectively. Transition radiation appears as a result of the interaction of pseudophotons with the conducting boundary.

surface give rise to a radiation that propagates into free space, away from the boundary. In the case of an ideal conductor, the transverse components of the radiation electric field can be obtained directly from Eq. (1):

$$
\begin{gathered}
E_{x, y}^{r}(z, x, \omega)=\frac{4 \pi i q}{v} e^{i k_{z} z} \frac{x_{x, y}}{x^{2}+\alpha^{2}}, \\
k_{z}=\sqrt{k^{2}-x^{2}}, \quad k=\omega / c .
\end{gathered}
$$

The $z$ component is found by using $\operatorname{div} \mathbf{E}^{r}=0$ that gives

$$
E_{z}^{r}=-\frac{\varkappa_{x} E_{x}^{r}+\chi_{y} E_{y}^{r}}{k_{z}} .
$$

Because at relativistic energies, which are those of practical interest, the longitudinal field component is considerably smaller than the transverse ones, for simplicity, it is not further taken into account in this paper.

By superimposing all plane waves of Eq. (2), one obtains the radiation field as a function of space coordinates

$$
E_{x, y}^{r}(z, \boldsymbol{\rho}, \omega)=\frac{i q}{\pi v} \int d x \frac{x_{x, y}}{x^{2}+\alpha^{2}} e^{i k_{z} z} e^{i x \boldsymbol{\rho}} .
$$

Here $\boldsymbol{\rho}$ is the radial vector lying in the $x, y$ plane, so that $x=\rho \cos \phi$ and $y=\rho \sin \phi$. After integration over angle $\psi$, Eq. (4) can be written in the form

$$
\begin{aligned}
& E_{x, y}^{r}(z, \boldsymbol{\rho}, \omega)=-\frac{2 q k}{v}\left\{\begin{array}{c}
\cos \phi \\
\sin \phi
\end{array}\right\} \zeta(\infty, 0, k z, k \rho), \\
& \zeta\left(\tau_{h}, \tau_{l}, w, u\right)=\int_{\tau_{l}}^{\tau_{h}} d t \frac{t^{2}}{t^{2}+(\beta \gamma)^{-2}} e^{-i w \sqrt{1-t^{2}}} J_{1}(u t) .
\end{aligned}
$$

In Eq. (5), $J_{1}$ is the Bessel function of the first kind and the function $\zeta$ of dimensionless variables entirely defines the radiation field evolution in space. At $w=0,|\zeta|^{2}$ can be considered the source intensity distribution. Formally the integral over $t$ must be taken from zero to infinity. In this case,

$$
|\zeta(\infty, 0,0, k \rho)|^{2}=\left|\frac{K_{1}(k \rho / \beta \gamma)}{\beta \gamma}\right|^{2},
$$

where $K_{1}$ is the modified Bessel function. The expression on the right-hand side of Eq. (6) is known as the Weizsäcker-Williams distribution of pseudophotons. Therefore Eq. (6) treats the OTR source as a bunch of pseudophotons and transition radiation simply appears as a reflection of the charge field. On the other hand, as directly follows from Eq. (5), the waves with $t>1$ are rapidly attenuated as the distance from the origin increases. These waves correspond to short distance static fields and do not give an appreciable contribution to the formation of the image. The value $t=1(x=k)$ is the maximum achievable for real photons.

It is a well-known concept that free fields originated by discontinuities in the motion of charged particles, or in the dielectric properties of media, need to travel for a distance called the "formation zone" before being completely "disentangled" from the particle field and acquiring all of the properties of a radiation field. This is exactly what happens in our case. The most used definition of the formation zone is

$$
l=\lambda / 2 \pi(1-\beta \cos \theta) .
$$

For ultrarelativistic particles this length can be very large along the particle path, but for a backward radiation it is of the order of $\lambda / 4 \pi$.

Figure 2 shows the field radial distributions $|\zeta(\infty, 0, k z, k \rho)|$ and $|\zeta(1,0, k z, k \rho)|$ at different distances from the origin plane (propagation path). It is evident that within less than a wavelength the two distributions become identical, i.e., within the formation zone the pseudophoton field transforms into a real photon field, and this transformation eliminates the discontinuity at $\rho \rightarrow 0$.

Excluding the short range field, we can conclude that the effective source distribution is determined by $|\zeta(1,0,0, k \rho)|^{2}$. The resolution provided by this distribution can be considered in a natural way as the intrinsic one. In reality, the diffraction phenomena produced by every finite optical system introduce limits that considerably modify this value.

\section{PROPAGATION THROUGH AN OPTICAL SYSTEM}

When the OTR source is properly defined, in order to find its image it is necessary to calculate the OTR field transmission through the elements of the optical system. 


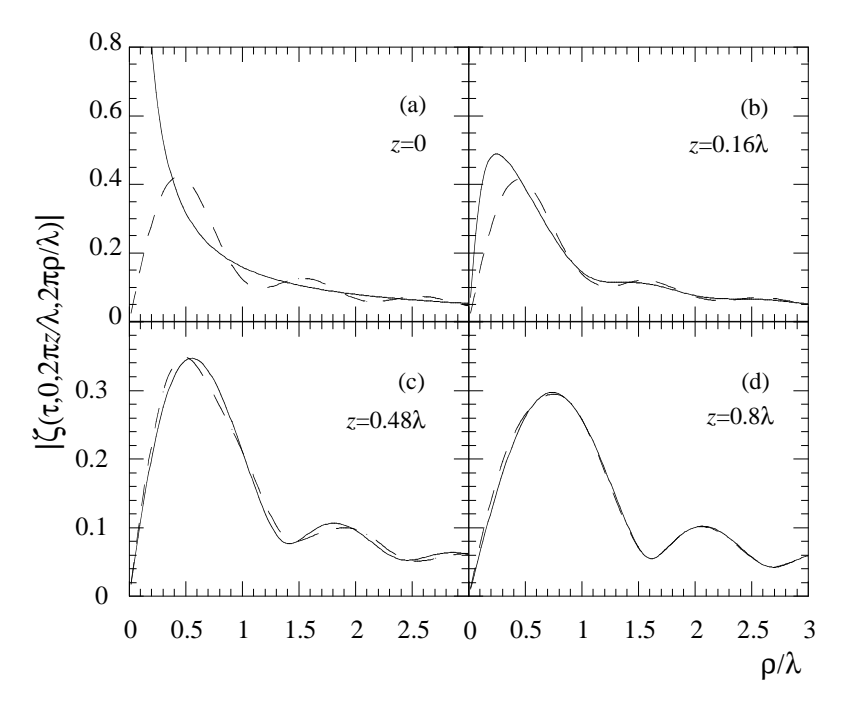

FIG. 2. The dimensionless function $\zeta$ represents the normalized radial distribution of the OTR field in the backward direction at different distances $z$ from the plane of origin for $\beta \gamma=1000$. The radial coordinate is given in units of the wavelength. Solid lines correspond to $\tau=\infty$ and dashed ones to $\tau=1$.

To do this we follow the ordinary technique [9] based on classical Kirchoff diffraction theory. For the sake of simplicity, let us approximate the optical system by a thin abberation-free lens located at distances $a$ and $b$ from the source and image planes (Fig. 3).

We will first find the field on the lens surface nearest to the source. In principle it can be deduced directly from Eq. (5); however, it is more convenient for this purpose to apply the well-known integral theorem of Helmholtz and Kirchoff [10]

$$
U_{-}\left(P_{l}\right)=\frac{1}{4 \pi} \int_{s}\left[V \frac{\partial}{\partial \mathbf{n}}\left(\frac{e^{i k r^{\prime}}}{r^{\prime}}\right)-\frac{e^{i k r^{\prime}}}{r^{\prime}} \frac{\partial V}{\partial \mathbf{n}}\right] d s .
$$

Here scalar field $U_{-}\left(P_{l}\right)$ stands for one of the OTR field components at an arbitrary point $P_{l}$ on the lens face,

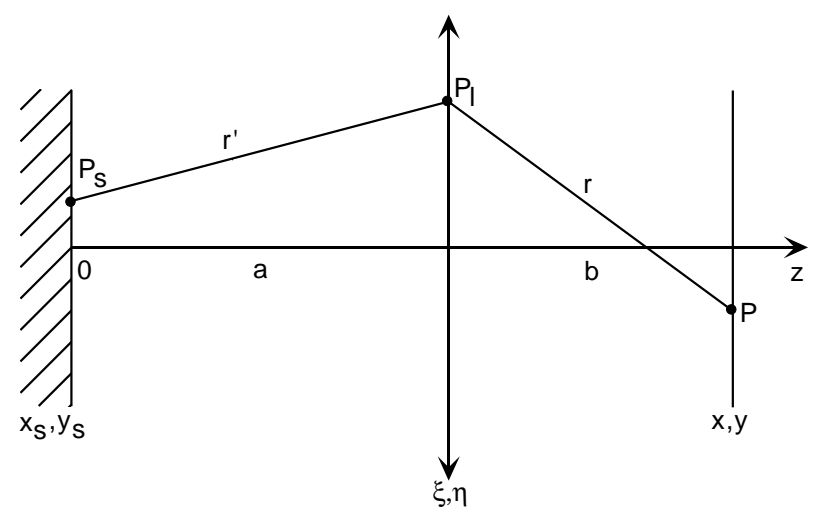

FIG. 3. Image of the OTR source is formed by a thin lens placed at distances $a$ and $b$ from the source plane $\left(x_{s}, y_{s}\right)$ and the image plane $(x, y)$. which, according to the theorem, can be obtained from the known field on the surface $s$. Considering $s$ the conductor boundary, the function $V$ in the right-hand side of Eq. (8) is exactly represented by the $x$ or $y$ field components of Eq. (4), $\mathbf{n}$ is the unit vector normal to this boundary, and $\mathbf{r}^{\prime}$ is the vector from an arbitrary point $P_{s}\left(x_{s}, y_{s}\right)$ on the boundary to the point $P_{l}(\xi, \eta)$, so that $r^{\prime}=\left|\mathbf{r}^{\prime}\right|$ is the distance between these two points. The integral in Eq. (8) is actually calculated across the whole boundary surface. As indicated in Fig. 3, three different sets of coordinates $\left(x_{s}, y_{s}\right),(\xi, \eta)$, and $(x, y)$ are used for the source, lens, and image planes, respectively. Substituting Eq. (4) computed at $z=0$ into Eq. (8), one can derive the following expression for $U_{-}\left(P_{l}\right)$ :

$$
\begin{aligned}
U_{-}\left(P_{l}\right)= & \frac{q k}{4 \pi^{2} v} \int d \boldsymbol{\rho}_{s} \int d \boldsymbol{x} \frac{x_{x, y}}{x^{2}+\alpha^{2}} \\
& \times e^{i \varkappa \rho_{s}} \frac{e^{i k r^{\prime}}}{r^{\prime}}\left(\frac{k_{z}}{k}+\hat{r}_{z}^{\prime}\right),
\end{aligned}
$$

where $\hat{r}^{\prime}=r^{\prime} / a$. Equation (9) shows explicitly that the field on the lens surface is built up from spherical waves emanating from every point of the source with amplitudes varying from point to point. Now we can use the standard simplification corresponding to the Fresnel approximation in the diffraction theory

$$
\frac{e^{i k r^{\prime}}}{r^{\prime}} \approx \frac{e^{i k a}}{a} e^{i(k / 2 a)\left[\left(\xi-x_{s}\right)^{2}+\left(\eta-y_{s}\right)^{2}\right]}
$$

The validity of Eq. (10) assumes that the distance between the source and the lens is large compared to their transversal dimensions and, therefore, we can neglect variation of the field amplitude along the wave front. With the same accuracy we can put $\hat{r}_{z}^{\prime}=a / r^{\prime} \approx 1$.

As is known, a thin lens simply introduces a phase delay that is a function of the coordinates on the lens surface

$$
h(\xi, \eta)=e^{-i(k / 2 f)\left(\xi^{2}+\eta^{2}\right)},
$$

where $f$ is the lens focal length. Hence the field on the lens face opposite to the source is

$$
U_{+}\left(P_{l}\right)=U_{-}\left(P_{l}\right) e^{-i(k / 2 f)\left(\xi^{2}+\eta^{2}\right)} .
$$

At this surface we can again apply the Kirchoff integral theorem, thus finding the field amplitude on the image plane. Under the assumption that the obliquity factor is equal to 2, and using a similar simplification as in Eq. (10), this gives, for an arbitrary point $P(x, y)$ of the image,

$$
\begin{aligned}
U(P) & =\frac{k}{2 \pi i} \int_{s_{l}} U_{+}\left(P_{l}\right) \frac{e^{i k r}}{r} d s_{l} \\
& \approx \frac{k}{2 \pi i} \frac{e^{i k b}}{b} \int_{s_{l}} U_{+}\left(P_{l}\right) e^{i(k / 2 b)\left[(\xi-x)^{2}+(\eta-y)^{2}\right]} d s_{l} .
\end{aligned}
$$

In Eq. (13) the integration is carried out over the lens aperture. Collecting the results throughout Eqs. (9)(13), omitting common phase factors not depending on 
integration variables and recalling that $U(P)$ refers to one of the OTR field components, we find

$$
\begin{aligned}
E_{x, y}(P, \omega)= & \frac{q}{(2 \pi)^{3} i} \frac{k^{2}}{a b v} \int d \boldsymbol{\rho}_{s} \\
& \times \int d x \frac{x_{x, y}}{x^{2}+\alpha^{2}} e^{i \varkappa \boldsymbol{\rho}_{s}} e^{i(k / 2 a) \rho_{s}^{2}}\left(\frac{k_{z}}{k}+1\right) \\
& \times \int d \xi d \eta e^{-i(k / a)\left[\xi\left(x_{s}+x / M\right)+\eta\left(y_{s}+y / M\right)\right]},
\end{aligned}
$$

where $M=b / a$ is the lens magnification. The last integral taken across the lens surface describes the effects of the optical system on the image due to diffraction from the finite lens aperture. For symmetry reasons, a cylindrical coordinate system is more convenient for further calculations. In addition to the notations in the source plane $x_{s}=\rho_{s} \cos \phi$ and $y_{s}=\rho_{s} \sin \phi$, we introduce similar ones in the image plane $x=\varrho M \cos \varphi$ and $y=\varrho M \sin \varphi$. Assuming the lens radius to be $d$, the diffraction pattern in Eq. (14) is easily evaluated

$$
\begin{array}{r}
\int d \xi d \eta e^{-i(k / a)\left[\xi\left(x_{s}+x / M\right)+\eta\left(y_{s}+y / M\right)\right]} \\
=\frac{2 \pi a d}{k p} J_{1}\left(\frac{k d}{a} p\right), \\
p=\sqrt{\rho_{s}^{2}+\varrho^{2}+2 \rho_{s} \varrho \cos (\phi-\varphi)} .
\end{array}
$$

Equation (14) then becomes

$$
\begin{aligned}
E_{x, y}(P, \omega)= & \frac{q}{(2 \pi)^{2} i} \frac{k d}{b v} \int d \boldsymbol{\rho}_{s} \int d x \frac{x_{x, y}}{x^{2}+\alpha^{2}} \\
& \times e^{i \boldsymbol{\varkappa} \boldsymbol{\rho}_{s}} e^{i(k / 2 a) \rho_{s}^{2}}\left(\frac{k_{z}}{k}+1\right) \frac{J_{1}(k d p / a)}{p} .
\end{aligned}
$$

\section{SINGLE PARTICLE OTR IMAGE}

As can be seen from Eq. (16), except for the exponential factor $e^{i(k / 2 a) \rho_{s}^{2}}$ and the multiplicand in parentheses that will be shown not to affect the image appreciably, Eq. (16) is nothing other than the convolution of the OTR source radial distribution with the well-known diffraction limited image distribution of a point source [the so-called point spread function (PSF)]. After trivial integration over $\psi$,

$$
\int_{0}^{2 \pi} d \psi\left\{\begin{array}{c}
\cos \psi \\
\sin \psi
\end{array}\right\} e^{i x \rho_{s} \cos (\psi-\phi)}=2 \pi i\left\{\begin{array}{c}
\cos \phi \\
\sin \phi
\end{array}\right\} J_{1}\left(x \rho_{s}\right),
$$

the evaluation of the integral over $\phi$ can be done by using an addition theorem on Bessel functions. Omitting intermediate steps, the final result is

$$
\begin{gathered}
\int_{0}^{2 \pi} d \phi\left\{\begin{array}{l}
\cos \phi \\
\sin \phi
\end{array}\right\} \frac{J_{1}(k d p / a)}{p} \\
=\frac{1}{\delta}\left\{\begin{array}{l}
\cos \varphi \\
\sin \varphi
\end{array}\right\} \sum_{m=1}^{\infty} R_{m} \frac{J_{2 m}(\delta \varrho)}{\varrho} \frac{J_{2 m}\left(\delta \rho_{s}\right)}{\rho_{s}}, \\
R_{m}=-8 \pi m, \quad \delta=\frac{k d}{a} .
\end{gathered}
$$

Using Eqs. (17) and (18), Eq. (16) can be reduced to

$$
\begin{aligned}
E_{x, y}(P, \omega)= & \frac{q}{2 \pi v} \frac{1}{M}\left\{\begin{array}{c}
\cos \varphi \\
\sin \varphi
\end{array}\right\} \int \frac{x^{2} d x}{x^{2}+\alpha^{2}}\left(\frac{k_{z}}{k}+1\right) \\
& \times \sum_{m=1}^{\infty} R_{m} \frac{J_{2 m}(\delta \varrho)}{\varrho} \\
& \times \int d \rho_{s} e^{i(k / 2 a) \rho_{s}^{2}} J_{1}\left(x \rho_{s}\right) J_{2 m}\left(\delta \rho_{s}\right) .
\end{aligned}
$$

A few remarks should be made here. As was already noted, in accordance with Kirchoff's principle the integration over $\rho_{s}$ is extended over the whole surface of the source. Therefore this approach based on diffraction theory allows, in a simple way, to take into account the finite size of the OTR target, in contrast to [6], where infinite transverse dimension of the conducting boundary was implicitly assumed. Meanwhile, as was recently established in [11], influence of the target finite size on the OTR spectral and angular characteristics is observable when the target extension is smaller in magnitude than $\lambda \gamma$. This value is often thought of as the particle field characteristic transverse size. For a long wavelength radiation, this effect is important already at medium energies. Particularly, it can play an important role in bunch length measurements by means of coherent transition radiation. In the case of visible light, it becomes noticeable for extremely high beam energies $\left(\gamma \geq 10^{5}\right)$. In the following we will assume $\gamma \ll 10^{5}$ and, therefore, consider that the conductor has infinite dimensions in the $x_{s}, y_{s}$ plane.

The exponential factor in Eq. (19) introduces corrections due to the spherical form of the wave front and the extension of the OTR source. It noticeably differs from unity in the region

$$
\rho_{s} \geq \sqrt{\lambda a} .
$$

Taking $\rho_{s}=\lambda \gamma$ as the characteristic size of the OTR source, we can always approximate the exponent with 1 whenever

$$
\lambda \gamma^{2} \ll a,
$$

thus neglecting the sphericity of the wave front or, equivalently, utilizing Fraunhofer's approximation. Even if the condition in Eq. (21) is not satisfied, significant effects are expected only at the periphery of the image, while, as follows from Fig. 2, the radiation intensity peaks in the spatial region extending over a few $\lambda$ 's around the axis. Thus the corrections produced by this exponential factor are generally small and Fraunhofer type diffraction is the main contribution to the image formation. A similar 
conclusion was derived in [6], but without adequate justification.

With the above assumptions, the integral over $\rho_{s}$ in Eq. (19) can be evaluated analytically

$$
\begin{aligned}
& \int_{0}^{\infty} d \rho_{s} J_{1}\left(x \rho_{s}\right) J_{2 m}\left(\delta \rho_{s}\right) \\
& = \begin{cases}\frac{x}{\delta^{2}} m_{2} F_{1}\left(1-m, 1+m ; 2 ; \frac{x^{2}}{\delta^{2}}\right), & \frac{\delta^{2}}{x^{2}}>1 \\
0, & \frac{\delta^{2}}{x^{2}}<1\end{cases}
\end{aligned}
$$

where ${ }_{2} F_{1}$ is the hypergeometric function.

When discussing the OTR source properties in the first section, we noticed that the limitation $x \leq 1$ on the possible values of the transverse wave vector component appears as the consequence of the transformation of the charge field into a radiation field. From Eq. (22), the effect of the finite aperture of the optics introduces a new limit $x<\delta$ that, under normal conditions, is more stringent.

Taking the result of the integration given by Eq. (22), the sum in Eq. (19) can be calculated using the Neuman series that is written as [12]

$$
\begin{aligned}
\left(\frac{l z}{2}\right)^{\mu-\nu} J_{\nu}(l z)= & l^{\mu} \sum_{n=0}^{\infty} \frac{\Gamma(\mu+n)}{n ! \Gamma(\nu+1)}(\mu+2 n) \\
& \times{ }_{2} F_{1}\left(-n, \mu+n ; \nu+1 ; l^{2}\right) J_{\mu+2 n}(z), \\
& \mu, \nu, \mu-\nu \neq-1,-2, \ldots
\end{aligned}
$$

Setting $n=m-1, \mu=2, \nu=1, l=x / \delta$, and $z=$ $\delta \varrho$, the summation over $m$ in Eq. (19) results in

$$
\begin{aligned}
\frac{8 \pi \varkappa}{\delta^{2} \varrho} \sum_{m=1}^{\infty} J_{2 m}(\delta \varrho) m_{2}^{2} F_{1}(1-m, 1 & \left.+m ; 2 ; \frac{\varkappa^{2}}{\delta^{2}}\right) \\
& =2 \pi J_{1}(x \varrho) .
\end{aligned}
$$

Collecting all of the results together and making the substitution $x=k t$, we obtain

$$
\begin{aligned}
E_{x, y}(P, \omega)= & -\frac{q k}{v M}\left\{\begin{array}{c}
\cos \varphi \\
\sin \varphi
\end{array}\right\} \int_{0}^{\theta_{\text {lens }}} \frac{t^{2} d t}{t^{2}+(\beta \gamma)^{-2}} \\
& \times\left(\sqrt{1-t^{2}}+1\right) J_{1}(k \varrho t),
\end{aligned}
$$

where $\theta_{\text {lens }}=d / a$ is the angular acceptance of the lens. Under real experimental conditions, it is always $\theta_{\text {lens }} \ll 1$ and one can consider with a good accuracy the factor in parentheses to be equal to 2 . Keeping in mind that according to the definition $\varrho=\rho / M$, where $\rho=\sqrt{x^{2}+y^{2}}$, the final result can be written as follows:

$$
E_{x, y}(P, \omega)=-\frac{2 q k}{v M}\left\{\begin{array}{c}
\cos \varphi \\
\sin \varphi
\end{array}\right\} \zeta\left(\theta_{\text {lens }}, 0,0, k \rho / M\right) .
$$

Equation (26) specifies the OTR field at the image point $P(\rho, \varphi)$. The intensity distribution in the image plane is given by the Poynting vector

$$
S(\rho, \omega)=\frac{c}{4 \pi^{2}}\left(\left|E_{x}\right|^{2}+\left|E_{y}\right|^{2}\right)
$$

that defines the radiant energy flux through the unit surface element per unit frequency interval. Equation (26) was derived for a single particle, and we can call the distribution of Eq. (27), calculated using the fields of Eq. (26), a single particle function (SPF), in analogy with the PSF defined for a point source.

If one now considers a bunch of particles, as long as coherent effects are negligible, the total beam profile image is the convolution of the SPF with the real transverse distribution of the particles in the bunch. The function $\zeta$ in Eq. (26) was used previously to describe the OTR source spatial distribution. By comparing Eqs. (5) and (26), one can conclude that the image distribution is the same as that of the source except for the magnification factor in the denominator and the upper limit of integration in $\zeta$.

The above expression for the SPF is consistent with that derived in [6]. Such a conclusion is a logical consequence of the simplifications used in the course of the analysis for the purpose of arriving at analytical expressions. Equation (25) is, in fact, obtained under the same assumptions made in [6], the most important of which are the ultrarelativistic regime and the applicability of the Fraunhofer diffraction regime.

The analysis performed shows clearly that Eq. (26) provides the main part of the image intensity distribution under normal conditions. Other effects, such as those due to the OTR source extension, finite dimensions of the OTR target, spherical character of the wave front, etc., can be considered higher order corrections and can be easily obtained numerically from the more complete expression [Eq. (19)].

For weakly relativistic (or nonrelativistic) regimes, most of the simplifications used are not justified. More accurate calculations are needed, starting from the exact formula of Eq. (9). Furthermore, a similar analysis for the longitudinal field component must also be performed in this case.

In the highly relativistic regime, we can conclude that the central part of the OTR SPF is energy independent (as far as $\theta_{\text {lens }} \gg \gamma^{-1}$ ) and defined by the diffraction on the lens, thus depending only on the lens aperture and the radiation wavelength. The spatial resolution given by the FWHM value of SPF is about 3 times larger than that for the standard PSF. Figure 4 shows the central part of the image for different values of the lens aperture. It was already observed in [6,7] that in defining the width of the OTR intensity distribution, the energy sensitive long tail can produce some problems. It was pointed out that by using the rms definition this tail leads to a large value of the final resolution. As a means to reduce the tail effect on spatial resolution, it was proposed to use a round opaque mask placed in the back focal plane of the lens to 


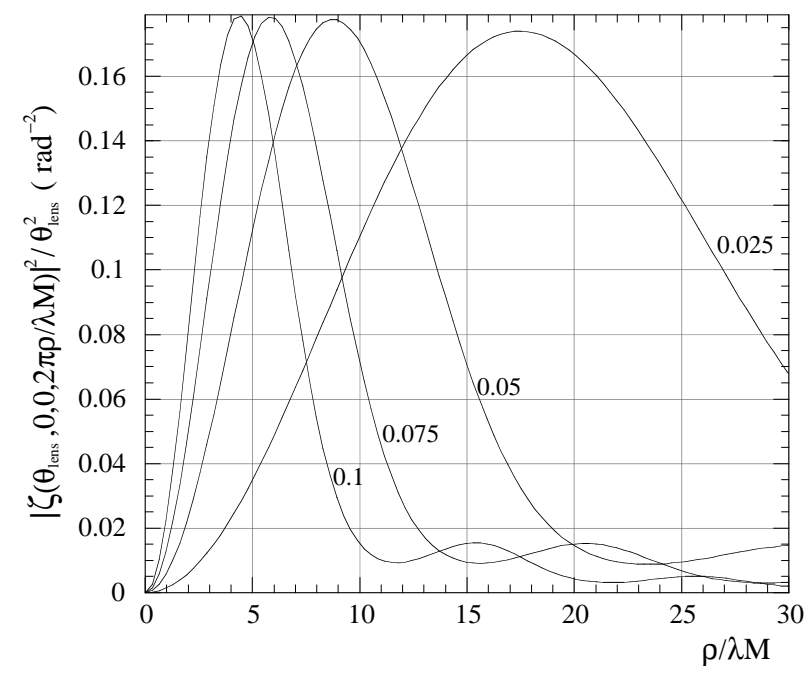

FIG. 4. Normalized OTR intensity distributions in the image plane (in units of $\theta_{\text {lens }}^{2}$ ) for different lens angular acceptances $\theta_{\text {lens }}(\beta \gamma=1000)$. Numbers on the curves are values of $\theta_{\text {lens }}$. Spatial coordinates in this figure, as well as in the following figures, are expressed in units of $\lambda M$, i.e., the wavelength increased by a factor of the lens magnification.

prevent the passage of photons at angles smaller than the mask angular acceptance $\theta_{\text {mask }}$.

As follows from our analysis, in the Fraunhofer regime, the effect of the lens aperture on the image is to revise the integration upper limit in the function $\zeta$ depending on the value of this aperture. This means simply that the lens accepts only photons emitted at angles less than $\theta_{\text {lens }}$; the presence of the mask thus introduces a new constraint on the photon angle. From this we can immediately conclude that the effect of the mask is to increase the lower limit of the integral for $\zeta$

$$
E_{x, y}(P, \omega)=-\frac{2 q k}{v M}\left\{\begin{array}{c}
\cos \varphi \\
\sin \varphi
\end{array}\right\} \zeta\left(\theta_{\text {lens }}, \theta_{\text {mask }}, 0, k \rho / M\right) .
$$

Here $\theta_{\text {mask }}=r / f$ is the angular radius of the mask image as seen from the object plane, while $r$ is the physical radius of the mask. When the mask is large, so that $\theta_{\text {mask }} \gg \gamma^{-1}$, one can derive the approximate expression for $\zeta$

$$
\begin{aligned}
\zeta\left(\theta_{\text {lens }}, \theta_{\text {mask }}, 0, u\right) & =\int_{\theta_{\text {mask }}}^{\theta_{\text {lens }}} \frac{t^{2} d t}{t^{2}+(\beta \gamma)^{-2}} J_{1}(u t) \\
& \approx \frac{J_{0}\left(u \theta_{\text {mask }}\right)-J_{0}\left(u \theta_{\text {lens }}\right)}{u} .
\end{aligned}
$$

Figure 5 gives an idea of the effect of the mask on the intensity distribution in the tail region. In the figure, thick solid lines plot the function $\left|K_{1}(u / \beta \gamma) / \beta \gamma\right|^{2}$ that, according to Eq. (6), is defined as $\lim \left|\zeta\left(\theta_{\text {lens }}, 0,0, u\right)\right|^{2}$ for $\theta_{\text {lens }} \rightarrow \infty$. The dashed lines correspond to another extreme case that follows from the first one when $\beta \gamma \rightarrow$ $\infty$, namely, $|\zeta|^{2}=1 / u^{2}$. It is worthwhile to remember that $K_{1}(u / \beta \gamma) / \beta \gamma$ describes the particle pseudophoton

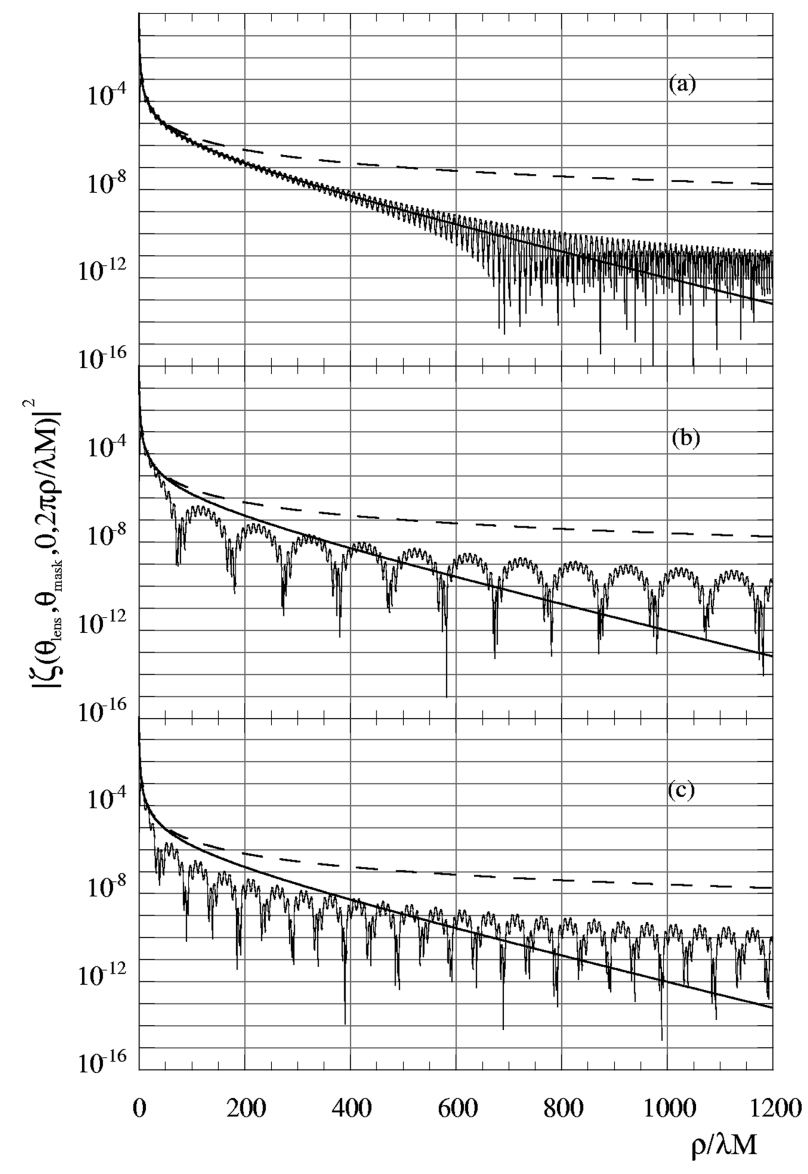

FIG. 5. Normalized image intensity distributions at the tail for $\theta_{\text {mask }}=0$ (a), $\theta_{\text {mask }}=0.05 \theta_{\text {lens }}$ (b), and $\theta_{\text {mask }}=0.1 \theta_{\text {lens }}$ (c), where $\theta_{\text {lens }}=0.1(\beta \gamma=1000)$. Reference curves in all subplots are the pseudophoton distribution $\left|K_{1}(u / \beta \gamma) / \beta \gamma\right|^{2}$ (thick solid lines) and the curve $1 / u^{2}$ (dashed lines), where $u$ stands for $\rho / \lambda M$.

field that for $u \ll \beta \gamma$ decreases as $1 / u$, while for $u>$ $\beta \gamma$ it falls more rapidly following an exponential law. It is a well-accepted fact that the region $u<\beta \gamma$ is to be considered the characteristic dimension of the particle field. The two curves are given as references to allow an easy comparison between the subplots in Fig. 5.

Figure 5(a) shows the "undisturbed" case $\theta_{\text {mask }}=0$. The intensity tail reveals the oscillatory behavior, in general, repeating the pseudophoton distribution. The period and amplitude of oscillations depend on the value of $\theta_{\text {lens }}$. Figures 5(b) and 5(c) refer to $\theta_{\text {mask }}=0.05 \theta_{\text {lens }}$ and $\theta_{\text {mask }}=0.1 \theta_{\text {lens }}$, respectively, for $\theta_{\text {lens }}$ equal to 0.1 and $\beta \gamma=1000$. Both curves are calculated from Eq. (29) and roughly follow the $1 / u^{2}$ dependence modulated by the difference of the two Bessel functions. For $\theta_{\text {mask }} \gg \beta \gamma^{-1}$, the tail intensity becomes $\gamma$ independent and is defined entirely by interference of the diffraction effects from the mask border and lens aperture leading to fringes in the intensity. An improvement at the tail is clearly achieved and the higher the beam energy the greater the benefit of using the mask. In this case it is 
also possible to calculate the total power

$I(\omega)=\int_{0}^{\infty} 2 \pi \rho d \rho S(\rho, \omega) \approx \frac{2}{\pi} \frac{q^{2} c}{v^{2}} \ln \frac{\theta_{\text {lens }}}{\theta_{\text {mask }}}$,

and we can conclude that the reduction in the tail intensity produced by the mask occurs at the expense of the total collected power.

\section{EXPERIMENTAL FACTORS AND OTR SPATIAL RESOLUTION}

The most frequent use of OTR in beam diagnostics is for the measurement of the transverse beam profile, because its high resolution and linearity make it superior to other imaging devices. The time properties of OTR also allow fast time resolved measurements.

In this context, the usual definition of the instrument resolution, as the minimum beam size that can be measured, is, unfortunately, not unique. The standard deviation $\sigma$ of a Gaussian distribution, the FWHM, and the second order momentum (rms) are among the most used definitions of the beam width under different experimental conditions. While for a defined analytical distribution, for example, a Gaussian, the relations among these quantities are well known, the same is not true for a generic distribution, and the tails, in particular, can produce large deviations.

The beam profile is normally analyzed projecting the beam image on the $x$ and $y$ planes. This procedure, first introduced in connection with nonimaging instruments such as secondary emission monitor (SEM) grids and scanning wires, has some solid foundation in the fact that motion in these two planes is normally uncoupled and that highly asymmetric beams are often used. Furthermore, the effects of statistical fluctuations in the beam distribution are reduced so that smoother beam profiles are obtained.

In order to investigate the resolution obtainable by OTR in this case, we can project Eq. (27) on the $x$ axis

$$
I_{p}(x, \omega)=\int S\left(\sqrt{x^{2}+y^{2}}, \omega\right) d y .
$$

Because of the symmetry of the distribution, an equivalent result is obtained when projecting on any other axis across the center of the image. To compute the above integral, one has to define the region over which the integration must be performed. In principle, the energy dependent long tail exhibited by the OTR image requires extending the integration to infinity, but a number of practical reasons prevents us from doing so. In particular,

(i) the sensitivity of the detector, and its thermal noise, define an absolute lower limit to the measurable intensity.

(ii) The digitalization of the image introduces a threshold in the minimum detectable intensity. A standard frame grabber for video rate signals has a dynamic range of 8 bits, so that intensities less than $0.4 \%$ of the saturation level cannot be detected. Using a slow digitizing device, coupled to a cooled detector to reduce the thermal noise, one can lower this limit, but not below $\approx 0.1 \%$ of saturation.

(iii) A diffuse background is almost always present, produced by external sources or by the beam itself. The best procedure for subtracting this background obviously depends on the particular case, but the subtraction always limits the lowest detectable tail intensity.

A standard approach [7] consists in finding the ellipse that contains a given fraction (depending on the background level) of the total intensity, typically of the order of $90 \%-95 \%$, and analyzing the projections of only the points inside the ellipse. Although this cut is somewhat arbitrary, it has the advantage of giving more weight to the core of the beam, the most interesting because the diffuse halo does not usually survive transport through complex optical systems.

For all of the above reasons, a cut in the OTR tail distribution can be safely introduced without significant loss of accuracy with respect to any real situation.

Figure 6 shows a 3D plot of the single particle OTR intensity distribution together with its projection obtained with a cutoff at $0.5 \%$ of the maximum intensity. Compared to the radial SPF in Fig. 4, the projection does not reproduce the deep minimum at the center, has about the same FWHM, and has a tail intensity more than twice as large.
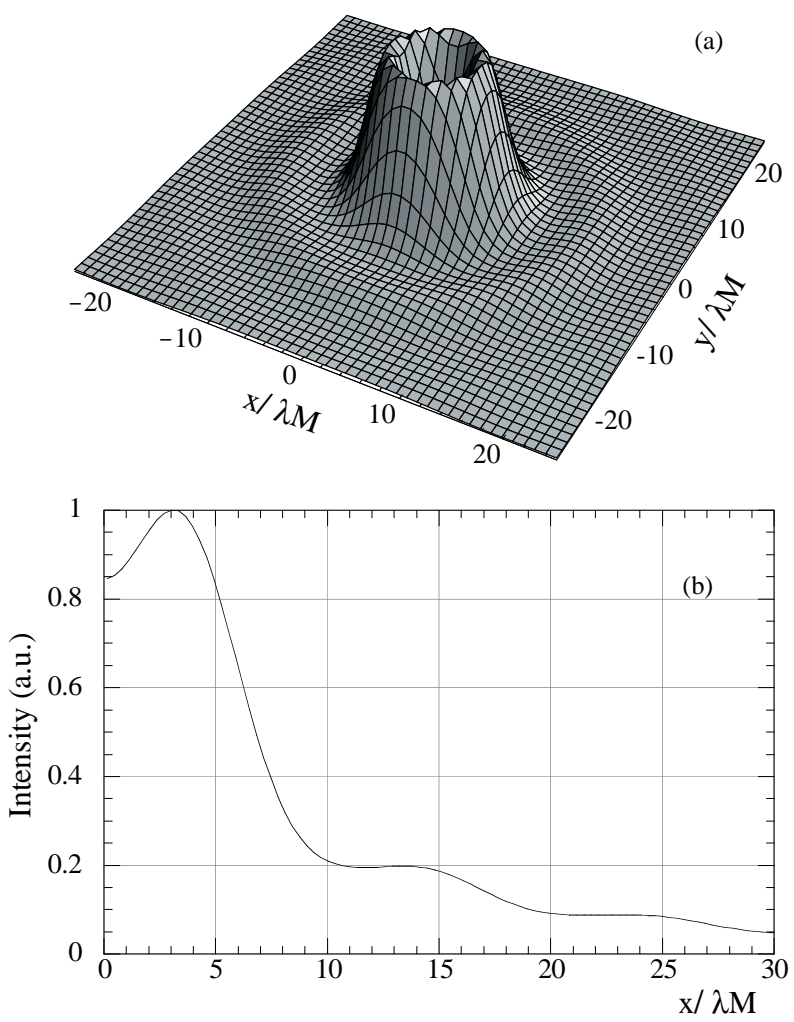

FIG. 6. 3D plot of the radial SPF (a) and its projection on the $x$ axis (b) for $0.5 \%$ cutoff level. The vertical scale in the $3 \mathrm{D}$ plot is the intensity in arbitrary units. $\theta_{\text {lens }}=0.1$ and $\beta \gamma=1000$. 

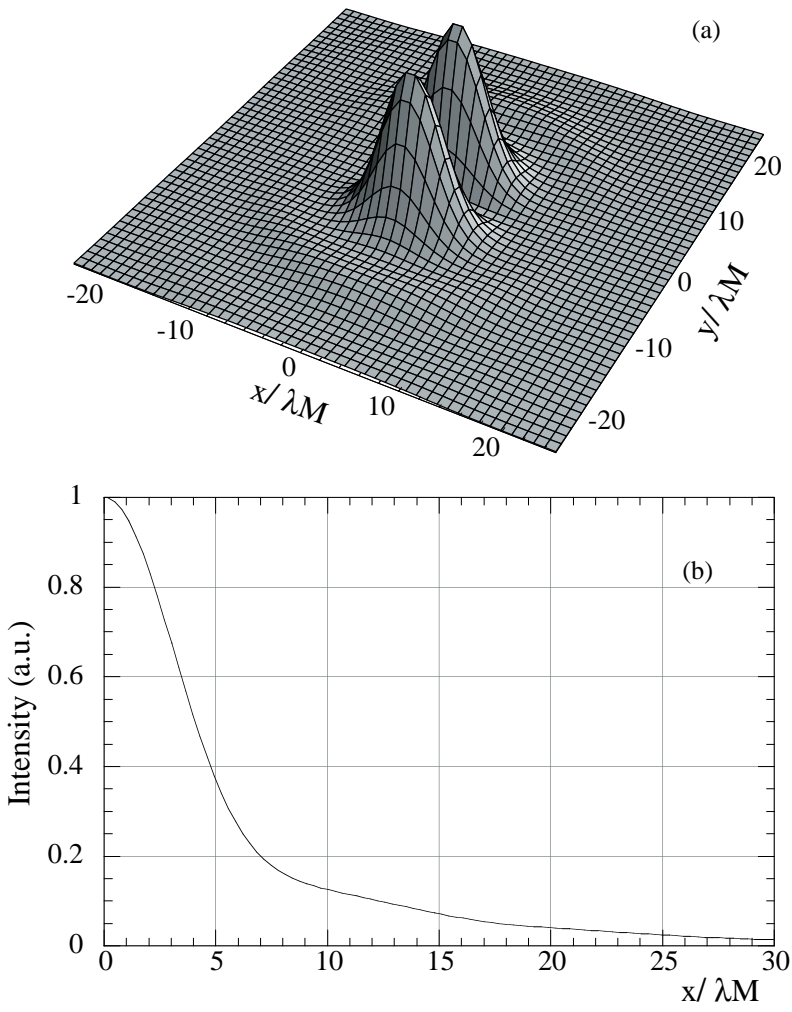

FIG. 7. 3D plot of the radial SPF for the $y$-polarized component (a) and its projection on the $x$ axis (b) for $0.5 \%$ cutoff level. The vertical scale in the 3D plot is the intensity in arbitrary units. $\theta_{\text {lens }}=0.1$ and $\beta \gamma=1000$.

A narrower distribution can be obtained by exploiting the OTR property of being radially polarized and selecting only the $x$ or $y$ component of the field by inserting a polarizer in the optical path. Figure 7 shows the 3D plot of the vertically polarized component of the radiation and the horizontally projected distribution with the same $0.5 \%$ cutoff. An identical distribution for the vertical plane is produced by the component polarized in the horizontal direction. In this case, the FWHM is almost two times smaller than that of the radial distribution, while the tail remains the same on average.

In Table I, the FWHM values of radial and projected distributions for both normal "unpolarized" and polarized cases are summarized for different beam energies and tail cutoff levels. Table II gives rms values of the distributions for the same energy and tail cutoff levels. It is evident that the FWHM values are rather constant, while the rms values are strongly dependent from both the energy and the tail cutoff, as was already anticipated in [7]. For a fixed cutoff level, there exists a corresponding energy range above which the growth of rms widths for all distributions saturates. Such a behavior demonstrates that the OTR spatial resolution with the rms beam width definition for high energy beams is determined by the cutoff, which, in turn, is dictated by experimental factors and, hence, is unavoidable.

For all of these considerations, it is clear that a simple definition of the minimum spatial resolution obtainable with OTR is not possible. As already pointed out in [7], when deriving an experimental beam transverse dimension from an OTR image, the standard rms size definition still maintains the quality of being shape independent, but the value is strongly affected by the background subtraction procedure.

On the contrary, the FWHM is a well-defined value for the single particle distribution, but applied to the beam size it can hide relevant aspects of beam shape. On the other hand, different definitions of resolution can be successfully applied to particular experimental situations. The more general approach consists in deriving from Eqs. (27) and (31) the SPF for the radial and projected distributions, respectively, and in using these functions to deconvolve the experimental measurement, obtaining the true beam profile, through a rather cumbersome numerical computation.

\section{CONCLUSION}

We have obtained a realistic description of the spatial distribution of the OTR source and used Kirchoff diffraction theory to find the intensity distribution produced by an individual particle in the image plane of a thin lens. At ultrarelativistic energies, our result is consistent with that found in [6]. In the frame of the model, one can also consider a number of other effects, for example, corrections to the Fraunhofer approximation and the influence of finite dimensions of the target. With the same accuracy, this approach allows one to make an image calculation for the case when charge passes through an opening in a conducting screen, e.g., circular hole or slits. Radiation produced herein is known as diffraction radiation and was shown [13] to be a possible tool for beam diagnostics.

We have demonstrated that the image is strongly dominated by the diffraction effects produced by the finite

TABLE I. FWHM values for radial SPF and projected ones in both normal and polarized cases for different particle energies. Numbers separated by slashes in each column correspond to $0.1 \%, 0.05 \%$, and $0.01 \%$ cutoff levels, respectively.

\begin{tabular}{lcccc}
\hline \hline & $E_{q}=0.1 \mathrm{GeV}$ & $E_{q}=0.5 \mathrm{GeV}$ & $E_{q}=1 \mathrm{GeV}$ & $E_{q}=5 \mathrm{GeV}$ \\
\hline Radial & $7.01 / 7.01 / 7.01$ & $7.08 / 7.08 / 7.08$ & $7.08 / 7.08 / 7.08$ & $7.08 / 7.08 / 7.08$ \\
Proj. & $6.55 / 6.56 / 6.57$ & $6.78 / 6.81 / 6.85$ & $6.79 / 6.84 / 6.89$ & $6.80 / 6.85 / 6.91$ \\
Proj. (pol). & $3.84 / 3.86 / 3.87$ & $4.05 / 4.08 / 4.14$ & $4.06 / 4.12 / 4.18$ & $4.07 / 4.14 / 4.20$ \\
\hline \hline
\end{tabular}


TABLE II. rms values for the same distributions, energies, and cutoff levels as in Table I.

\begin{tabular}{lcccc}
\hline \hline & $E_{q}=0.1 \mathrm{GeV}$ & $E_{q}=0.5 \mathrm{GeV}$ & $E_{q}=1 \mathrm{GeV}$ & $E_{q}=5 \mathrm{GeV}$ \\
\hline Radial & $7.29 / 7.78 / 8.32$ & $9.27 / 10.8 / 13.6$ & $9.43 / 11.3 / 15.5$ & $9.49 / 11.5 / 16.8$ \\
Proj. & $7.67 / 8.75 / 10.4$ & $10.9 / 13.2 / 22.2$ & $11.2 / 15.3 / 27.3$ & $11.2 / 15.7 / 30.8$ \\
Proj. (pol). & $5.43 / 6.19 / 7.32$ & $7.72 / 9.35 / 15.7$ & $7.89 / 10.8 / 19.3$ & $7.94 / 11.1 / 21.8$ \\
\hline \hline
\end{tabular}

dimension of the optical system and that the peculiar structure of the OTR image causes some problem in the definition of spatial resolution. While in principle the rms width should increase with beam energy, real experimental factors impose cutting the intensity tails off, thus saturating the energy dependence. On the contrary, the FWHM is substantially independent from both energy and tail intensity cutoffs and is only a few times larger than the standard PSF.

The use of a mask in the back focal plane of the optical system, to cut off the radiation at small angles, reduces the tail level at the price of a smaller total intensity.

By selecting only one component of the radiation polarization, a better resolution in one plane can be obtained, approaching that of SPF.

[1] L. Wartski, S. Roland, J. Lasalle, M. Bolore, and G. Filippi, J. Appl. Phys. 46, 3644 (1975).

[2] D. W. Rule, Nucl. Instrum. Methods Phys. Res., Sect. B 24/25, 901 (1987).

[3] D. W. Rule and R. B. Fiorito, in Accelerator Instrumentation, edited by Elliott S. McCrory, AIP Conf. Proc. No. 229 (AIP, New York, 1991), p. 315.
[4] M. Castellano, M. Ferrario, S. Kulinski, M. Minestrini, P. Patteri, F. Tazzioli, L. Catani, L. Gregorini, and S. Tazzari, Nucl. Instrum. Methods Phys. Res., Sect. A 357, 231 (1995).

[5] J.-C. Denard, P. Piot, K. Capek, and E. Feldi, in Proceedings of the 17th IEEE Particle Accelerator Conference (PAC-97), Vancouver, Canada, 1997 (to be published).

[6] V. A. Lebedev, Nucl. Instrum. Methods Phys. Res., Sect. A 372, 344 (1996).

[7] X. Artru, M. Castellano, L. Catani, R. Chehab, D. Giove, K. Honkavaara, P. Patteri, F. Tazzioli, M. Taurigna-Quere, A. Variola, and L. Wartski, Laboratoire de l'Accelerateur Lineaire Report No. 97-30, 1997.

[8] V.L. Ginzburg and V. N. Tsytovich, Transition Radiation and Transition Scattering (Adam Hilger, Bristol, 1990).

[9] A. K. Ghatak and K. Thyagarajan, Contemporary Optics (Plenum, New York, 1978).

[10] M. Born and E. Wolf, Principles of Optics (Pergamon, New York, 1965).

[11] N.F. Shul'ga and S. N. Dobrovol'skii, Pis'ma Zh. Eksp. Teor. Fiz. 65, 581 (1997) [JETP Lett. 65, 611 (1997)].

[12] H. Buchholz, The Confluent Hypergeometric Function (Springer-Verlag, New York, 1969), p. 130.

[13] M. Castellano, Nucl. Instrum. Methods Phys. Res., Sect. A 394, 275 (1997). 